\author{
Accelerator Division \\ Alternating Gradient Synchrotron Department \\ BROOKHAVEN NATIONAL LABORATORY \\ Upton, New York 11973 \\ Accelerator Division \\ Technical Note \\ AGS/AD/Tech. Note No. 387

\section{RESONANCE EXCITATION IN THE AGS BOOSTER \\ DUE TO CLOSED ORBIT DISTORTION IN \\ MULTIPOLE MAGNETS}

\author{
C.J. Gardner
}

January 19, 1994 


\title{
Resonance Excitation in the AGS Booster Due to Closed Orbit Distortion in Multipole Magnets
}

\author{
C. J. Gardner
}

January 19, 1994

\section{Introduction}

A number of studies $[1,2]$ were carried out in 1993 to determine the corrections required to reduce or eliminate beam loss due to excitation of transverse resonances in the AGS Booster. During the course of these. studies it was found that the quadrupole corrections could not completely eliminate the beam loss observed as the $2 Q_{x}=9$ and $2 Q_{y}=9$ resonances were crossed. It was thought initially that an $18 \mathrm{th}$ harmonic octupole field might be responsible for the residual loss, but then it was found that the required corrections varied linearly with orbit radius which strongly suggested that quadrupole fields arising from the displacement of the closed orbit in sextupole fields were exciting the resonances. Beam particles whose momentum differs by $\delta p$ from the central momentum oscillate obout orbits which are displaced in the sextupoles and therefore see a quadrupole field which is proportional to $\delta p$. If the sextupole field possesses a ninth harmonic, or if some combination of orbit harmonics and sextupole harmonics produce a ninth harmonic, then the quadrupole-driven resonances can be excited and this can account for the residual loss. It was found that by introducing a ninth harmonic with available sextupoles, the dependence of the required quadrupole correction on radius could be eliminated and the residual loss could be reduced substantially.

Further studies and careful analysis by Y. Shoji $[2,3]$ showed that the skew quadrupole and sextupole corrections required to eliminate beam loss as the $Q_{x}+Q_{y}=9$ and $3 Q_{x}=14$ resonances are crossed also depend linearly on the orbit radius. This again suggested that the displacement of the closed orbit in higher order multipole fields-in this case skew sextupole 
and octupole fields-gives rise to lower order multipoles (skew quadrupole and sextupole) which excite the lower order resonances. The purpose of this note is to work out the details of how the displacement of the closed orbit in higher order multipoles gives rise to lower order multipoles which excite lower order resonances. The treatment is an extension of that given in Ref.[4] for the case of closed orbit distortions in sextupoles. We shall assume that the reference orbit lies in a plane and shall employ the right-handed curvilinear coordinate system ( $x, y, s)$ introduced in Ref.[5].

\section{The Multipole Vector Potential}

The hamiltonian treatment given in subsequent sections requires expressions for the vector potential of a multipole magnet. Here we develop these and other formulae for use later on.

Inside a multipole magnet, far from the magnet ends, the magnetic field is transverse to the reference orbit and one can choose a gauge such that the vector potential has no transverse components. The $x$ and $y$ components of the magnetic field are then

$$
B_{x}=\frac{\partial A_{s}}{\partial y}, \quad B_{y}=-\frac{\partial A_{s}}{\partial x}
$$

where $A_{s}$ is the longitudinal component of the vector potential. Since the curl of the magnetic field is zero we then have

$$
\frac{\partial^{2} A_{s}}{\partial x^{2}}+\frac{\partial^{2} A_{s}}{\partial y^{2}}=0
$$

which has solutions

$$
A_{n}(z)=\frac{z^{n}}{n !}=\frac{1}{n !}(x+i y)^{n}=U_{n}(x, y)+i V_{n}(x, y)
$$

where $U_{n}$ and $V_{n}$ are real functions of $x$ and $y$. Differentiation of (3) with respect to $x$ and $y$ yields the Cauchy-Riemann equations

$$
\frac{\partial U_{n}}{\partial x}=\frac{\partial V_{n}}{\partial y}, \quad \frac{\partial U_{n}}{\partial y}=-\frac{\partial V_{n}}{\partial x}
$$

from which it follows that

$$
\frac{\partial^{2} U_{n}}{\partial x^{2}}+\frac{\partial^{2} U_{n}}{\partial y^{2}}=0, \quad \frac{\partial^{2} V_{n}}{\partial x^{2}}+\frac{\partial^{2} V_{n}}{\partial y^{2}}=0
$$


Thus both $U_{n}(x, y)$ and $V_{n}(x, y)$ are real solutions of (2) and we call them respectively the normal and skew $2 n$-pole vector potentials. Expanding $(x+i y)^{n}$ in (3) we find the following normal and skew vector potentials:

Dipole:

$$
U_{1}=x, \quad V_{1}=y
$$

Quadrupole:

$$
U_{2}=\frac{1}{2}\left(x^{2}-y^{2}\right), \quad V_{2}=x y
$$

Sextupole:

$$
U_{3}=\frac{1}{6}\left(x^{3}-3 x y^{2}\right), \quad V_{3}=-\frac{1}{6}\left(y^{3}-3 x^{2} y\right)
$$

Octupole:

$$
U_{4}=\frac{1}{24}\left(x^{4}-6 x^{2} y^{2}+y^{4}\right), \quad V_{4}=\frac{1}{24}\left(4 x^{3} y-4 x y^{3}\right)
$$

Decapole:

$$
U_{5}=\frac{1}{120}\left(x^{5}-10 x^{3} y^{2}+5 x y^{4}\right), \quad V_{5}=\frac{1}{120}\left(5 x^{4} y-10 x^{2} y^{3}+y^{5}\right)
$$

Differentiation of (3) with respect to $x$ and $y$ also yields the relations

$$
\frac{\partial U_{n}}{\partial x}=U_{n-1}, \quad \frac{\partial V_{n}}{\partial x}=V_{n-1}, \quad \frac{\partial U_{n}}{\partial y}=-V_{n-1}, \quad \frac{\partial V_{n}}{\partial y}=U_{n-1} \text {. }
$$

Expanding $(z+d)^{n}$, where $d=a+i b$, we obtain

$$
\frac{(z+d)^{n}}{n !}=\frac{z^{n}}{n !}+\frac{d z^{n-1}}{(n-1) !}+\frac{d^{2} z^{n-2}}{2 !(n-2) !}+\cdots+\frac{d^{n-1} z}{(n-1) !}+\frac{d^{n}}{n !}
$$

which may be written as

$$
\begin{aligned}
A_{n}(z+d) & =A_{n}(z)+d A_{n-1}(z)+\frac{1}{2 !} d^{2} A_{n-2}(z)+\cdots \\
& +\frac{1}{2 !} z^{2} A_{n-2}(d)+z A_{n-1}(d)+A_{n}(d)
\end{aligned}
$$


$\bullet$

•

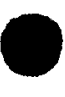


Expressing each $A_{m}$ as $U_{m}+i V_{m}$ we then have

$$
\begin{aligned}
U_{n}(x+a, y+b) & =U_{n}(x, y)+a U_{n-1}(x, y)-b V_{n-1}(x, y) \\
& +\frac{1}{2}\left(a^{2}-b^{2}\right) U_{n-2}(x, y)-a b V_{n-2}(x, y)+\cdots \\
& +\frac{1}{2}\left(x^{2}-y^{2}\right) U_{n-2}(a, b)-x y V_{n-2}(a, b) \\
& +x U_{n-1}(a, b)-y V_{n-1}(a ; b)+U_{n}(a, b)
\end{aligned}
$$

and

$$
\begin{aligned}
V_{n}(x+a, y+b) & =V_{n}(x, y)+a V_{n-1}(x, y)+b U_{n-1}(x, y) \\
& +\frac{1}{2}\left(a^{2}-b^{2}\right) V_{n-2}(x, y)+a b U_{n-2}(x, y)+\cdots \\
& +\frac{1}{2}\left(x^{2}-y^{2}\right) V_{n-2}(a, b)+x y U_{n-2}(a, b) \\
& +x V_{n-1}(a, b)+y U_{n-1}(a, b)+V_{n}(a, b)
\end{aligned}
$$

\section{Hamiltonian for Oscillations about the Reference Trajectory}

The hamiltonian for oscillations about the reference trajectory is [6]

$$
H=-(1+h x)\left\{1-\left(P_{x}-U\right)^{2}-\left(P_{y}-V\right)^{2}\right\}^{1 / 2}-(1+h x) W
$$

where

$$
U=\frac{e A_{x}}{c p}, \quad V=\frac{e A_{y}}{c p}, \quad W=\frac{e A_{s}}{c p} ; \quad P_{x}=\frac{p_{x}}{p}, \quad P_{y}=\frac{p_{y}}{p},
$$

$A_{x}, A_{y}, A_{s}$ are the components of the vector potential, $p_{x}$ and $p_{y}$ are the components of the momentum along $x$ and $y$, and $p$ is the particle momentum. The momentum of the reference particle is $p_{0}$. We shall assume that the effects of any longitudinal magnetic fields (typically near the ends of magnets) can be neglected, in which case the transverse components of the vector potential may be set to zero. The longitudinal component of the vector potential is then

$$
A_{s}=A y-B x+B h x^{2} / 2-B_{1}\left(x^{2}-y^{2}\right) / 2+C_{n} F_{n}(x, y)
$$


.

•

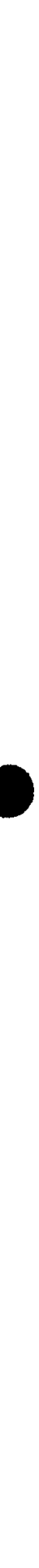

○ 
where $A, B$, and $B_{1}$ are the values of $B_{x}, B_{y}$, and $\partial B_{y} / \partial x$ along the reference trajectory,

$$
h=\frac{e B_{0}}{c p_{0}},
$$

and $B_{0}$ is the value of the vertical guide field for which a particle of momentum $p_{0}$ follows the reference trajectory. The departure of $B$ from $B_{0}$ and of $A$ from 0 are the errors in the vertical and horizontal fields along the reference trajectory. $C_{n} F_{n}(x, y)$ is the vector potential for the multipole under consideration.

Thus we have

$$
W=\frac{p_{0}}{p}\left\{g y-\frac{B}{B_{0}}\left(h x-\frac{1}{2} h^{2} x^{2}\right)+\frac{1}{2} n h^{2}\left(x^{2}-y^{2}\right)+K_{n} F_{n}(x, y)\right\},
$$

where

$$
g=\frac{e A}{c p_{0}}, \quad n h^{2}=-\frac{e B_{1}}{c p_{0}}, \quad K_{n}=\frac{e C_{n}}{c p_{0}},
$$

and the hamiltonian is then

$$
H=H_{0}+H_{n},
$$

where

$$
\begin{aligned}
H_{0} & =\frac{1}{2}\left(P_{x}^{2}+P_{y}^{2}\right)+\frac{1}{2} \frac{p_{0}}{p}\left(\frac{B}{B_{0}}-n\right) h^{2} x^{2}+\frac{1}{2} \frac{p_{0}}{p} n h^{2} y^{2} \\
& +\frac{B p_{0}-B_{0} p}{B_{0} p} h x-\frac{p_{0}}{p} g y-\frac{p_{0}}{p} h g x y
\end{aligned}
$$

and

$$
H_{n}=-\frac{p_{0}}{p} K_{n} F_{n}(x, y) .
$$

Here we have omitted the term proportional to $h x F_{n}(x, y)$ in $H_{n}$, and only terms to second order in coordinates and momenta have been retained in the expression for $H_{0}$. The $n h^{2} x^{2}$ and $n h^{2} y^{2}$ terms in (23) are responsible for horizontal and vertical focusing, and the factor $p_{0} / p$ multiplying these terms gives rise to the variation of the tunes with momentum. The $h x$ term is responsible for horizontal dispersion and for the distortion of the horizontal closed orbit due to errors in the guide field. Likewise the $g y$ term is responsible for the distortion of the vertical closed orbit. The hgxy term couples the oscillations in the horizontal and vertical planes whenever the curvature, $h$, and the horizontal field are nonzero $(g \neq 0)$ along the 
-

•

- 
reference trajectory. (This could occur if, for example, the horizontal bending magnets were rolled by some amount about the longitudinal axis thereby producing a horizontal field in a region of nonzero curvature.) Note that by setting $p=p_{0}, B=B_{0}$, and $g=0$ in (23) one obtains

$$
H_{0}=\frac{1}{2}\left(P_{x}^{2}+P_{y}^{2}\right)+\frac{1}{2}(1-n) h^{2} x^{2}+\frac{1}{2} n h^{2} y^{2}
$$

which is the usual hamiltonian for linear oscillations about the reference trajectory.

The equations of motion, obtained from the canonical equations

$$
x^{\prime}=\frac{\partial H}{\partial P_{x}}, \quad P_{x}^{\prime}=-\frac{\partial H}{\partial x}, \quad y^{\prime}=\frac{\partial H}{\partial P_{y}}, \quad P_{y}^{\prime}=-\frac{\partial H}{\partial y}
$$

are

$$
x^{\prime \prime}+\frac{p_{0}}{p}\left(\frac{B}{B_{0}}-n\right) h^{2} x+\frac{B p_{0}-B_{0} p}{B_{0} p} h-\frac{p_{0}}{p} h g y-\frac{p_{0}}{p} K_{n} \frac{\partial F_{n}}{\partial x}=0,
$$

and

$$
y^{\prime \prime}+\frac{p_{0}}{p} n h^{2} y-\frac{p_{0}}{p} g(1+h x)-\frac{p_{0}}{p} K_{n} \frac{\partial F_{n}}{\partial y}=0
$$

Defining

$$
\delta p=p-p_{0}, \quad \delta B=B-B_{0}
$$

we have

$$
\frac{p_{0}}{p}=1-\frac{\delta p}{p}, \quad \frac{B}{B_{0}}=1+\frac{\delta B}{B_{0}},
$$

and, to first order in $\delta p / p$ and $\delta B / B_{0}$,

$$
\frac{p_{0}}{p}\left(\frac{B}{B_{0}}-n\right)=\left(1-\frac{\delta p}{p}\right)(1-n)+\frac{\delta B}{B_{0}}, \quad \frac{B p_{0}-B_{0} p}{B_{0} p}=\frac{\delta B}{B_{0}}-\frac{\delta p}{p} .
$$

Equations (27-28) then become

$$
\begin{aligned}
x^{\prime \prime} & +\left(1-\frac{\delta p}{p}\right)(1-n) h^{2} x+\frac{\delta B}{B_{0}} h^{2} x+\left(\frac{\delta B}{B_{0}}-\frac{\delta p}{p}\right) h \\
& -\left(1-\frac{\delta p}{p}\right) h g y-\left(1-\frac{\delta p}{p}\right) K_{n} \frac{\partial F_{n}}{\partial x}=0
\end{aligned}
$$

and

$$
y^{\prime \prime}+\left(1-\frac{\delta p}{p}\right) n h^{2} y-\left(1-\frac{\delta p}{p}\right) g(1+h x)-\left(1-\frac{\delta p}{p}\right) K_{n} \frac{\partial F_{n}}{\partial y}=\dot{0}
$$


•

- 


\section{The Distorted Closed Orbit}

The deviation of the distorted closed orbit from the reference trajectory is given by the periodic solutions, $x_{c}(s)$ and $y_{c}(s)$, of equations (32) and (33). The lowest-order solutions are

$$
x_{c}(s)=\frac{\delta p}{p} D(s)+d_{x}(s), \quad y_{c}(s)=d_{y}(s)
$$

where

$$
\begin{gathered}
D^{\prime \prime}+(1-n) h^{2} D-h=0, \quad d_{x}^{\prime \prime}+(1-n) h^{2} d_{x}+\frac{\delta B}{B_{0}} h=0 \\
d_{y}^{\prime \prime}+n h^{2} d_{y}-g=0 .
\end{gathered}
$$

Here $D(s)$ is the horizontal dispersion and $d_{x}(s)$ and $d_{y}(s)$ are the distortions of the horizontal and vertical closed orbits due to dipole errors. Thus both the horizontal dispersion and horizontal dipole errors contribute to $x_{c}$ while only vertical dipole errors contribute to $y_{c}$.

\section{Hamiltonian for Oscillations about the Distorted Closed Orbit}

Following Ref.[4] we introduce new coordinates and momenta

$$
q_{1}=x-x_{c}, \quad p_{1}=P_{x}-x_{c}^{\prime}, \quad q_{2}=y-y_{c}, \quad p_{2}=P_{y}-y_{c}^{\prime},
$$

which represent the deviations of the particle trajectory from the distorted closed orbit. This transformation is canonical and is generated by

$$
F_{2}\left(x, p_{1}, y, p_{2}\right)=\left(x-x_{c}\right)\left(p_{1}+x_{c}^{\prime}\right)+\left(y-y_{c}\right)\left(p_{2}+y_{c}^{\prime}\right) .
$$

Thus we have

$$
\begin{array}{ll}
P_{x}=\frac{\partial F_{2}}{\partial x}=p_{1}+x_{c}^{\prime}, & q_{1}=\frac{\partial F_{2}}{\partial p_{1}}=x-x_{c} \\
P_{y}=\frac{\partial F_{2}}{\partial y}=p_{2}+y_{c}^{\prime}, & q_{2}=\frac{\partial F_{2}}{\partial p_{2}}=y-y_{c}
\end{array}
$$

and the new hamiltonian is

$$
G=H+\frac{\partial F_{2}}{\partial s}=H-x_{c}^{\prime}\left(p_{1}+x_{c}^{\prime}\right)+q_{1} x_{c}^{\prime \prime}-y_{c}^{\prime}\left(p_{2}+y_{c}^{\prime}\right)+q_{2} y_{c}^{\prime \prime} .
$$


-

-

- 
Using (23-24) and (27-28) in (41) we find

$$
G=G_{0}+G_{n}
$$

where

$$
\begin{aligned}
& G_{0}=\frac{1}{2}\left(p_{1}^{2}+p_{2}^{2}\right)+\frac{1}{2} \frac{p_{0}}{p}\left(\frac{B}{B_{0}}-n\right) h^{2} q_{1}^{2}+\frac{1}{2} \frac{p_{0}}{p} n h^{2} q_{2}^{2}-\frac{p_{0}}{p} h g q_{1} q_{2}, \\
& G_{n}=-\frac{p_{0}}{p} K_{n}\left[F_{n}\left(q_{1}+x_{c}, q_{2}+y_{c}\right)-q_{1} \frac{\partial F_{n}}{\partial x}\left(x_{c}, y_{c}\right)-q_{2} \frac{\partial F_{n}}{\partial y}\left(x_{c}, y_{c}\right)\right]
\end{aligned}
$$

Using (11) and (14-15) in (44) we find, for the case in which $F_{n}=U_{n}$,

$$
\begin{aligned}
G_{n} & =-\frac{p_{0}}{p} K_{n}\left[U_{n}\left(q_{1}, q_{2}\right)+x_{c} U_{n-1}\left(q_{1}, q_{2}\right)-y_{c} V_{n-1}\left(q_{1}, q_{2}\right)\right. \\
& \left.+\frac{1}{2}\left(x_{c}^{2}-y_{c}^{2}\right) U_{n-2}\left(q_{1}, q_{2}\right)-x_{c} y_{c} V_{n-2}\left(q_{1}, q_{2}\right)+\cdots\right]
\end{aligned}
$$

and for the case in which $F_{n}=V_{n}$,

$$
\begin{aligned}
G_{n} & =-\frac{p_{0}}{p} K_{n}\left[V_{n}\left(q_{1}, q_{2}\right)+x_{c} V_{n-1}\left(q_{1}, q_{2}\right)+y_{c} U_{n-1}\left(q_{1}, q_{2}\right)\right. \\
& \left.+\frac{1}{2}\left(x_{c}^{2}-y_{c}^{2}\right) V_{n-2}\left(q_{1}, q_{2}\right)+x_{c} y_{c} U_{n-2}\left(q_{1}, q_{2}\right)+\cdots\right]
\end{aligned}
$$

Here we see that the displacements, $x_{c}$ and $y_{c}$, of the closed orbit in the multipoles $U_{n}$ and $V_{n}$ give rise to lower order normal and skew multipoles which are proportional to $x_{c}, y_{c}, x_{c}^{2}, y_{c}^{2}$, and $x_{c} y_{c}$. Using the approximate expressions (34) for $x_{c}$ and $y_{c}$ we obtain the following hamiltonians for various multipoles.

Sextupole:

$$
G_{3}=-K_{3}\left[U_{3}+\frac{\delta p}{p} D U_{2}+d_{x} U_{2}-d_{y} V_{2}\right]+\cdots
$$

Skew Sextupole:

$$
G_{3}=-K_{3}\left[V_{3}+\frac{\delta p}{p} D V_{2}+d_{x} V_{2}+d_{y} U_{2}\right]+\cdots
$$

Octupole:

$$
G_{4}=-K_{4}\left[U_{4}+\frac{\delta p}{p} D U_{3}+d_{x} U_{3}-d_{y} V_{3}\right]+\cdots
$$


.

-

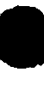


Skew Octupole:

$$
G_{4}=-K_{4}\left[V_{4}+\frac{\delta p}{p} D V_{3}+d_{x} V_{3}+d_{y} U_{3}\right]+\cdots
$$

Thus the strength of the normal (skew) $2(n-1)$-pole arising from the horizontal displacement of the closed orbit in a normal (skew) $2 n$-pole magnet is

$$
K(s)=-K_{n}(s)\left[\frac{\delta p}{p} D(s)+d_{x}(s)\right],
$$

and the strength of the skew (normal) $2(n-1)$-pole arising from the vertical displacement of the closed orbit in a normal (skew) $2 n$-pole magnet is

$$
K(s)= \pm K_{n}(s) d_{y}(s)
$$

\section{Resonance Excitation}

Transverse resonances [7] are defined by the equation

$$
m Q_{x}+n Q_{y}=N
$$

where $Q_{x}$ and $Q_{y}$ are the horizontal and vertical tunes, and $m, n$, and $N$ are integers. If $m$ and $n$ have opposite signs, the resonance is called a difference resonance; otherwise it is called a sum resonance. The order, $l$, of the resonance is

$$
l=|m|+|n| .
$$

The resonance condition (53) arises from the first-order perturbation

treatment of the vector potential terms $x^{|m|} y^{|n|}$ associated with the $2 l$-pole field. Resonance excitation can occur only if the tunes are sufficiently close to the resonance and the excitation coefficient,

$$
\kappa=\int_{0}^{2 \pi r} \beta_{x}^{|m / 2|} \beta_{y}^{|n / 2|} K(s) e^{i \psi} d s
$$

is nonzero. Here

$$
\begin{gathered}
\psi(s)=m\left(\mu_{x}-Q_{x} \theta\right)+n\left(\mu_{y}-Q_{y} \theta\right)+N \theta, \quad \theta=s / r \\
\mu_{x}(s)=\int_{0}^{s} \frac{d s^{\prime}}{\beta_{x}\left(s^{\prime}\right)}, \quad \mu_{y}(s)=\int_{0}^{s} \frac{d s^{\prime}}{\beta_{y}\left(s^{\prime}\right)}, \quad K(s)=\frac{e}{c p}\left(\frac{\partial^{(l-1)} B}{\partial x^{(l-1)}}\right),
\end{gathered}
$$




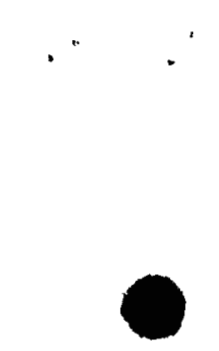

-

- 
and $B=B_{y}\left(B_{x}\right)$ if $n$ is even (odd). Since

$$
\psi(s)=m \mu_{x}+n \mu_{y} \approx N \theta,
$$

when the tunes are near the resonance, we see that the excitation coefficient is essentially the $N$ th harmonic, in azimuth $\theta$, of the multipole strength $K(s)$. Thus if the tunes are sufficiently close to the resonance, i.e. if they are within the resonance stopband, and if $n$ is even (odd), the resonance will be excited by the $N$ th harmonic of the normal (skew) $2 l$-pole fields present in the machine. The width of the stopband is proportional to the strength of the $2 l$-pole field, and for resonances of order 3 and higher also depends on the amplitudes of the betatron oscillations.

In the AGS Booster we therefore have the following possibilities:

The resonances $2 Q_{x}=9$ and $2 Q_{y}=9$ can be excited by the ninth harmonic present in

$$
K(s)=-K_{3}(s)\left[\frac{\delta p}{p} D(s)+d_{x}(s)\right],
$$

where $K_{3}(s)$ is the sextupole strength along the reference trajectory, or by the ninth harmonic present in

$$
K(s)=-K_{3}(s) d_{y}(s)
$$

where $K_{3}(s)$ is the skew sextupole strength along the reference trajectory. The resonance $Q_{x}+Q_{y}=9$ can be excited by the ninth harmonic present in

$$
K(s)=-K_{3}(s)\left[\frac{\delta p}{p} D(s)+d_{x}(s)\right],
$$

where $K_{3}(s)$ is the skew sextupole strength along the reference trajectory, or by the ninth harmonic present in

$$
K(s)=K_{3}(s) d_{y}(s),
$$

where $K_{3}(s)$ is the sextupole strength along the reference trajectory.

The resonances $3 Q_{x}=14$ and $Q_{x}+2 Q_{y}=14$ can be excited by the 14 th harmonic present in

$$
K(s)=-K_{4}(s)\left[\frac{\delta p}{p} D(s)+d_{x}(s)\right],
$$


where $K_{4}(s)$ is the octupole strength along the reference trajectory, or by the 14th harmonic present in

$$
K(s)=-K_{4}(s) d_{y}(s),
$$

where $K_{4}(s)$ is the skew octupole strength along the reference trajectory. The resonances $3 Q_{y}=14$ and $Q_{y}+2 Q_{x}=14$ can be excited by the 14 th harmonic present in

$$
K(s)=-K_{4}(s)\left[\frac{\delta p}{p} D(s)+d_{x}(s)\right],
$$

where $K_{4}(s)$ is the skew octupole strength along the reference trajectory, or by the 14th harmonic present in

$$
K(s)=K_{4}(s) d_{y}(s),
$$

where $K_{4}(s)$ is the octupole strength along the reference trajectory. The contributions of $D$ and $d_{x}$ to K(s) in the AGS Booster are discussed in Ref.[3].

\section{References}

1. C. Gardner and Y. Shoji, "Observation and Correction of Resonance Stopbands in the AGS Booster", Proceedings of the 1993 Particle Accelerator Conference, May 17-20, Washington, D.C.

2. Y. Shoji, AGS Studies Reports 282, 286-300, 305, 307, 308.

3. Y. Shoji, "Harmonic Analysis of the AGS Booster Imperfections", Proceedings of the Orbit Correction and Analysis Workshop, December 1-3, 1993, Brookhaven National Laboratory, Upton, New York.

4. R.D. Ruth, "Single-Particle Dynamics in Circular Accelerators", Physics of Particle Accelerators, AIP Conference Proceedings No. 153, American Institute of Physics, New York, 1987, pp. 181-183.

5. K.L. Brown and R.V. Servranckx, "First- and Second-Order Charged Particle Optics",Physics of High Energy Particle Accelerators, AIP Conference Proceedings No. 127, American Institute of Physics, New York, 1985, pp. 62-138. 
6. A.J. Dragt, "Lectures on Nonlinear Orbit Dynamics", Physics of High Energy Particle Accelerators, AIP Conference Proceedings No. 87, American Institute of Physics, New York, 1982, pp. 147-313.

7. G. Guignard, "The General Theory of all Sum and Difference Resonances in a Three-Dimensional Magnetic Field in a Synchrotron", CERN 76-06, 23 March 1976; "A General Treatment of Resonances in Accelerators" CERN 78-11, 10 November 1978. 